\title{
Clinico-Mycological Profile of Otomycosis in Chronic Suppurative Otitis Media Patients Attending a Tertiary Care Teaching Hospital
}

\author{
Sapna Chauhan ${ }^{1 *}$ (D) and Surender ${ }^{2}$ \\ ${ }^{1}$ Department of Microbiology, Muzaffarnagar Medical College, Muzaffarnagar - 251 203, Uttar Pradesh, India. \\ ${ }^{2}$ Department of Anaesthesia, Muzaffarnagar Medical College, Muzaffarnagar - 251 203, Uttar Pradesh, India.
}

\begin{abstract}
Chronic Suppurative Otitis Media (CSOM) is commonly encountered chronic inflammation of middle ear as well as the mastoid cavity due to dysfunction of Eustachian tube followed by microbial infection. Fungal infection in CSOM is now a major otolarynological problem in India not only in children but in adults too. Excessive use of steroids, antibiotics, cytotoxic chemotherapies and immunosuppressive diseases has increased the incidence of otomycosis in recent years. To define the aetiology of clinically diagnosed otomycosis. To isolate and identify fungal agents and their association with different factors (age, sex, predisposing factors). A total of $\mathbf{1 0 0}$ clinically diagnosed patients of CSOM with suspicion of otomycosis were included in the study. Patients where passing swab is difficult as with canal stenosis were excluded. Samples were taken using sterile swabs and studied for microbial profile. Male to female ratio in study was 1.6:1. The most common fungi isolated in CSOM cases was Aspergillus fumigatus followed by Aspergillus niger. Other fungus isolated were Aspergillus flavus, Penicillium species, Mucor species and Candida species. 04 samples showed mixed growth of Aspergillus species and Candida species. In our study we concluded that Aspergillus complex was most commonly isolated fungi in CSOM cases.
\end{abstract}

Keywords: CSOM, Otomycosis, Aspergillus spp

*Correspondence: drsapna_chauhan@yahoo.com
(Received: January 14, 2021; accepted: May 14, 2021)
Citation: Chauhan S, Surender. Clinico-Mycological Profile of Otomycosis in Chronic Suppurative Otitis Media Patients Attending
a Tertiary Care Teaching Hospital. J Pure Appl Microbiol. 2021;15(2):813-818. doi: 10.22207/JPAM.15.2.35
@ The Author(s) 2021. Open Access. This article is distributed under the terms of the Creative Commons Attribution 4.0 International License which
permits unrestricted use, sharing, distribution, and reproduction in any medium, provided you give appropriate credit to the original author(s) and
the source, provide a link to the Creative Commons license, and indicate if changes were made. 


\section{INTRODUCTION}

Otomycosis has a worldwide distribution. Its prevalence in CSOM cases can be high as $77 \%{ }^{1}$. However it is more prevalent in warm, humid climates as compared to arid or cold. Recent years have reported an increase in not only prevalence of otomycosis but also an increase in therapeutic failure. Excessive use of steroids, indiscriminate use of antibiotics, cytotoxic chemotherapies and immunosuppressive diseases has increased the incidence of otomycosis in recent years ${ }^{2}$.

Otomycosis in CSOM presents with non specific symptoms that include intractable itching, irritation, discomfort, pain and discharge from ear. Aspergillus and Candida species are the most common fungal genera responsible for otomycosis. Aspergillus alone accounts for $75 \%$ of cases with Aspergillus niger being the commonest followed by $A$. flavus and $A$. fumigatus ${ }^{3}$.

Several predisposing factors like increased and indiscriminate use of topical antibiotics,instillation of hot oil/water in the ear, unhygienic mopping of ear,use of hearing aids,swimming in contaminated water etc are associated with Otomycosis ${ }^{2}$.

The present study was undertaken with the aim of defining the aetiology of clinically diagnosed otomycosis, in CSOM patients, to isolate and identify fungal agents and also their association with different factors (age,sex, predisposing factors).

Table 1. Gender-wise distribution of study population

\begin{tabular}{lc}
\hline Sex & Percentage (\%) \\
\hline Male & 61 \\
Female & 39 \\
\hline
\end{tabular}

Table 2. Age-wise distribution of study population

\begin{tabular}{lc}
\hline Age group & Percentage(\%) \\
\hline $0-10$ & 0 \\
$11-20$ & 7 \\
$21-30$ & 45 \\
$31-40$ & 28 \\
$41-50$ & 9 \\
$51-60$ & 4 \\
61 and above & 7
\end{tabular}

\section{MATERIAL AND METHODS}

The study was conducted in the Department of Microbiology and Department of ENT of Muzaffarnagar Medical College. The study was initiated after obtaining approval from Institutional ethical committee. A total of 100 clinically diagnosed patients of CSOM were included in the study. Patients where passing swab is difficult as with canal stenosis were excluded. Demographic details age, sex, occupation and history of associated risk factors was collected from each study participant. History of Immunocompromised state like diabetes, pregnancy, HIV, autoimmune disorders etc was also recorded in the case recording form.

Samples were taken using sterile swabs. Three swabs were collected and transported immediately to the laboratory. One swab was used for Gram staining, second swab for direct examination of fungal elements using $10 \%$ potassium hydroxide and third swab was processed for fungal culture.

For fungal culture two sets of Sabouraud's dextrose agar were incubated at $37 \& 25^{\circ} \mathrm{C}$. These were examined for any growth everyday on first week and twice a week for next three weeks. Growth was observed for rate of growth,morphology of colonies, texture and surface pigmentation. Microscopic examination using Lacto phenol cotton blue and slide culture were done to identify the fungi. Gram staining, germ tube test were performed for Candida species. A negative fungal culture report was given after 4 weeks.

\section{RESULTS}

Age, Sex, Occupation and Socioeconomic Status: The study population consisted of $39 \%$

Table 3. Distribution of study population according to occupation

\begin{tabular}{lc}
\hline Occupation & Percentage(\%) \\
\hline Agricultural labourer & 27 \\
Housewife & 17 \\
Manual labourer & 20 \\
Retired & 6 \\
Student & 13 \\
Teacher & 9 \\
Sales Job & 8
\end{tabular}


Table 4. Distribution of study population according to predisposing factors

\begin{tabular}{lc}
\hline Predisposing factors & Percentage (\%) \\
\hline Use of hot oil/water in ear & 49 \\
Bath in river/pond & 9 \\
Use of topical antibiotics/steroids & 17 \\
Diabetes & 5 \\
Post mastoidectomy & 2 \\
Not significant & 18 \\
\hline
\end{tabular}

females and $61 \%$ males(Table 1 ). The highest incidence was in the age group of 21-30 years while none of the patient was reported in paediatric age group of 0-10 years(Table 2). Maximum number of male patients were involved in agricultural and other associated work. Most of the females were housewives (Table 3).

Side and laterality: Of study population, $39 \%$ had otomycosis of left ear, $53 \%$ of right ear and only $8 \%$ had bilateral otomycosis.

Predisposing factors: The most common predisposing factor in our study came out to be instillation of ear drops with $49 \%$ followed by use of topical antibiotics(17\%). (Table 4).Other predisposing features seen were Swimming in ponds/river, diabetes and post surgery (Table 4).

Microbiology: Amongst 100 patients 3 did not show any fungal growth. Aspergillus species was most common fungus isolated, seen in $69 \%$ cases. A. fumigatus complex was the commonest (37\%) followed by $A$. niger (20\%) and $A$. flavus (12\%). Candida species (18\%), Mucor species (2\%) and Penicillium (4\%) were other fungi isolated. 3 cases showed mixed infection by Candida spp and A.fumigatus while 1 case had mixed growth of Candida and A.niger (Table 5).

\section{DISCUSSION}

Incidence of fungal infections has shown an increasing trend in recent years. Increased number of immunocompromised patients and indiscriminate use of topical antibiotics renders individuals more susceptible to Otomycosis. Such patients land up in therapeutic failure to antibiotic treatment,hence knowing the causative fungi is desirable for effective treatment in all CSOM cases. In our study male to female patient ratio came out to be $1.6: 1$. The highest incidence of
Table 5. Different fungi isolated from study population

\begin{tabular}{lc}
\hline Fungus isolated & Percentage (\%) \\
\hline Aspergillus fumigatus & 37 \\
Aspergillus niger & 20 \\
Aspergillus flavus & 12 \\
Candida spp. & 18 \\
Mucor spp. & 2 \\
Penicillium spp. & 4 \\
Candida spp+ Aspergillus fumigatus & 3 \\
Candida spp+ Aspergillus niger & 1 \\
No growth & 3 \\
\hline
\end{tabular}

otomycosis in CSOM was seen in young males. This is in accordance with studies by many authors ${ }^{4}$, $5,6,7$. These young males were mainly involved in agricultural work and labour work. Highest incidence in these may be attributed to the fact that they are more exposed to saprophytic fungal spores as compared to elderly. Similar findings are also recorded in studies by various other authors ${ }^{8}$ 9,10 . Amongst females most of them(17\%) were housewives who are usually involved in household work. Housewives sweep the house/floor/garden and so may have more chances of being exposed to fungal spores.

In our study only $8 \%$ of individuals showed bilateral involvement which corresponds to studies by Ho et al and Aggarwal et al who found bilateral involvement in $7 \%$ and $7.89 \%$ cases $^{10,11}$. Unilateral involvement was as high as $92 \%$ which correlated with studies by Aggarwal et al and Paulose et $a l^{11,12}$.

The incidence of Otomycosis was seen highest in those individual who gave history of instillation of hot oil into the ear (49\%) followed by the use of topical antibiotics and steroids(17\%). This is in accordance to study done by Prasad et $a l^{2}$.

Lack of knowledge and belief in certain myths that application of hot oil(Coconut or mustard usually ) will be beneficial in curing ear problems may be the reason of increased in the incidence of Otomycosis.

Another important predisposing factor in our study came out to be the use of antibiotic drops/steroids for various ear ailments without valid medical consultation. Indiscriminate use of 
antibiotics disrupt the commensal flora of external auditory canal, may change the $\mathrm{pH}$ favouring growth of fungus.

Another important predisposing factor (9\%)came out to be swimming in pond/river. Swimming keeps the external auditory canal moist also removes the protective lipid layer lining the canal causing damage to the meatal wall thus favouring fungal growth. $5 \%$ patients in our study were diabetic. No other immunocompromised state or disease was identified in any of the study subjects. Thus there is a need to control the co morbid conditions as well so as to prevent recurrences and refractoriness to treatment.

$2 \%$ cases were seen post mastoidectomy. This is similar to study done by Pradhan et $a /$. Surgeries may favour fungal growth because of use of antibiotics and steroids,also surgeries disrupt the anatomy, cause meatal damage which may be favouring fungal growth.

Amongst 100 cases, 3 samples did not show any fungal growth. Self-medication with antifungal agents, improper sample collection and stringent growth requirements by certain fungus may prevent their growth on culture media.

Out of remaining 97 , the most commonly isolated organism was Aspergillus species (69\%) followed by Candida species (18\%). Amongst Aspergillus, A.fumigatus was isolated in $37 \%$, A.niger in $20 \%$ and $A$. flavus in $12 \%$ samples. Most of the studies worldwide ${ }^{13-17}$ and from India ${ }^{18-21}$ have showed similar findings of Aspergillus species being commonest followed by Candida species.

Although Aspergillus has been the common causative agents in most of the studies but its species differ in different studies. While in our study we report Aspergillus fumigatus to be the predominant species isolated, many studies report Aspergillus niger as most common. The distribution of fungi is affected geographically. Kaur et al have also reported Aspergillus fumigatus $(41.1 \%)$ to be most common isolated fungi followed by Aspergillus niger (36.9\%) ${ }^{9}$.

The other species isolated in our study were Candida species, Mucor species and Penicillium species. In a study by Prasad et al Aspergillus species was isolated in $80 \%$ of cases followed by Penicillium species (8\%), Candida albicans (4\%), Rhizopus species(1\%) and
Chrysosporium species (1\%)

4 samples in our study showed a mixed growth of two fungi. 3 samples showed growth of Aspergillus fumigatus and Candida species and 1 sample showed mixed growth of Aspergillus niger and Candida species. 6 cases in study by Prasad et al showed mixed growth of 2 fungi.3 had co-existing Aspergillus niger and $A$. fumigatus while 3 had Aspergillus niger and $A$.flavus ${ }^{2}$. Mixed infection of Aspergillus niger and Candida albicans was also reported in a study from Nigeria ${ }^{22}$.

Also in a study by Priti et al 32 samples showed mixed growth of 2 fungi. 14 had dual growth of Aspergillus niger and Candida albicans, 8 showed Aspergillus flavus and Aspergillus fumigatus, 6 Aspergillus niger and Aspergillus fumigatus and 4 Aspergillus flavus and Candida species $^{23}$. Mixed fungal infection though rare but are difficult to treat and usually occur in immunosuppressed individuals ${ }^{24}$. Therapeutic failure in these mixed infections is probably due to biofilm formation ${ }^{25-27}$.

\section{CONCLUSION}

Otomycosis in CSOM cases is increasing worldwide. This is an alarm to otologists as well as a need to promptly evaluate patients who did not respond to antibacterial treatment.

In our study young males were most (61\%) commonly affected population. It is usually a unilateral disease with $53 \%$ involvement of the right ear.

The incidence was seen high in individuals who gave history of instillation of hot oil into the ear (49\%) followed by the use of topical antibiotics and steroids (17\%).

The most commonly isolated etiological agent in cultures was Aspergillus species (69\%) followed by Candida species (18\%). Amongst Aspergillus, A.fumigatus was isolated in $37 \%$, A.niger in $20 \%$ and A.flavus in $12 \%$ samples.

Mixed infection of two fungi was also seen in 4 cases.

Thus we conclude that it is necessary to go for laboratory examination of swabs collected from CSOM cases for fungal culture especially when patient have been using topical steroids or antibiotics for long time. Laboratory examination would also help in finding out mixed infections 
which may otherwise be difficult to treat. Also educating people about myths and on the counter use of antibiotics should be stopped,only then Otomycosis cases in CSOM will be reduced.

\section{ACKNOWLEDGMENTS}

None.

\section{CONFLICT OF INTEREST}

The authors declare that there is no conflict of interest.

\section{AUTHORS' CONTRIBUTION}

All authors listed have made a substantial, direct and intellectual contribution to the work, and approved it for publication.

\section{FUNDING}

None

\section{DATA AVAILABILITY}

All datasets generated or analysed during this study are included in the manuscript and/or the Supplementary Files.

\section{ETHICS STATEMENT}

Not applicable

\section{REFERENCES}

1. Prakash R, Juyal D, Negi V, et al. Adekhandi S, Sharma M, Sharma N. Microbiology of chronic suppurative otitis media in a tertiary care setup of Uttarakhand state, India. N Am J Med Sci. 2013;5(4):282- 7. doi: 10.4103/1947-2714.110436

2. Prasad SC, Kotigadde S, Shekhar M, et al. Primary Otomycosis in the Indian Subcontinent:Predisposing Factors, Microbiology, and Classification. Int J Microbiol.2014;2014:636493. doi: 10.1155/2014/636493

3. Chander J. Textbook of Medical Mycology. 3rd Edition,New Delhi,Mehta Publishers; 2002.

4. Mugliston T, O'Donoghue G. Otomycosis-a continuing problem. Journal of Laryngology and Otology. 1985;99(4):327-333. doi: 10.1017/ S002221510009678X

5. Pradhan B, Tuladhar NR, Amatya RM. Prevalence of otomycosis in outpatient department of otolaryngology in Tribhuvan University Teaching Hospital, Kathmandu, Nepal. Annals of Otology, Rhinology and Laryngology. 2003;112(4):384-387. doi: 10.1177/000348940311200416

6. Viswanatha B, Sumatha D, Vijayashree MS. Otomycosis in immunocompetent and immunocompromised patients: comparative study and literature review. Ear, Nose \& Throat Journal. 2012;91:114-121. doi:

\subsection{7/014556131209100308}

7. Yehia MM, Al-Habib HM, Shehab NM. Otomycosis:a common problem in North Iraq. J Laryngol Otol. 1990;104(5):387-389. doi: 10.1017/ S0022215100158529

8. Than KM, Naing KS, Min M. Otomycosis in Burma and its treatment. Am J Trop Med Hyg. 1980; 29(4):620623. doi: $10.4269 /$ ajtmh.1980.29.620

9. Kaur R, Mittal N, Kakkar M, Aggarwal AK, Mathur MD. Otomycosis:A clinicomycologic study. Ear Nose Throat J. 2000; 79(8):606-609. doi: 10.1177/014556130007900815

10. Ho T, Vrabec JT, Yoo D, Coker NJ. Otomycosis:Clinical features and treatment implications. Otolaryngol Head Neck Surg. 2006;135: 787-791. doi: 10.1016/j. otohns.2006.07.008

11. Aggarwal SK,Jaiswal K. Fungal Profile and Its Characteristics in Patients of Otomycosis-A Prospective Study. National Journal Of Laboratory Medicine. 2019;8 (4):4-7. doi: 10.7860/NJLM/2019/42535:2366

12. Paulose KO, Al-Khalifa S, Shenoy P, Sharma RK. Mycotic infections of the ear (otomycosis):A prospective study. J laryngol Otol. 1989; 103(1):30-35. doi: 10.1017/ S0022215100107960

13. Pontes ZBVDS, Silva ADF, Lima EDO, et al. Otomycosis: a retrospective study. Braz J of Otorhinolaryngology. 2009;75(3):367-370. doi: 10.1590/S180886942009000300010

14. Stern JC,Lucente FE.Otomycosis. Ear, Nose and Throat Journal.1988;67(11)804-810.

15. Wadhwani K, Srivastava AK. Fungi from otitis media of agricultural field workers. Mycopathologia. 1984;88(2,3)155-159. doi: 10.1007/BF00436447

16. Barati B,Okhovvat SAR, Goljanian A,Omrani MR.Otomycosis in central Iran: a clinical and mycological study. Iranian Red Crescent Medical Journal. 2011;13(12):873-876.

17. Oliveri S, Capello G, Napolitano MG, Triolo C, Grillo C. Otomycosis:etiology and analysis of predisposing factors. Boll Ist Sieroter Milan. 1984;63 (6):537-542

18. Chander J, Maini S, Subrahmanyan S, Handa A .Otomycosisa-a clinico-mycological study and efficacy of mercurochrome in its treatment. Mycopathologia.1996;135 (1):9-12. doi: 10.1007/ BF00436569

19. Mohanty JC, Mohanty SK, Sahoo RC, et al. Clinicomicrobial profile of otomycosis in Berhampur. Indian Journal of Otology. 1999;5(2):81-83.

20. Agrawal SR, Jain AK, Goyal RB, Gupta A, Gupta KG. A Clinicomycological study of otomycosis with special reference to silent tympanic membrane perforation. Indian Journal of Otology. 2001;7(2):49-52.

21. Kumar A. Fungal spectrum in otomycosis patient. JK Science. 2005;7(3):152-155.

22. Gugnani HC, Okafor BC, Nzelibe F, Njoku-Obi AN. Etiological agents of otomycosis in Nigeria. Mycoses. 1989;32: 224-229. doi: 10.1111/j.1439-0507.1989. tb02236.x

23. Agarwal P, Devi LS. Otomycosis in a Rural Community Attending a Tertiary Care Hospital: Assessment of Risk Factors and Identification of Fungal and Bacterial Agents. J Clin Diagn Res. 2017;11(6):14-18. doi: 
$10.7860 / J C D R / 2017 / 25865.10068$

24. Soll DR. Mixed Mycotic Infections.In:Brogden KA,Guthmiller JM editors.Polymicrobial diseases. Washingto(DC):ASMPress;2002.Chapter 17.

25. Gregson AE, La Touche CJ. Otomycosis:a neglected disease. J Laryngol Otol. 1961;75: 45-69. doi: 10.1017/ S0022215100057467
26. Philip AA, Thomas R, Job A, Sundaresan VR, S. Anandan, Albert RR. Effectiveness of 7.5 percent povidone iodine in comparison to 1 percent clotrimazole with lignocaine in the treatment of otomycosis. ISRN Otolaryngology. 2013;2013:239730. doi: 10.1155/2013/239730

27. Murray PA. Manual of Clinical Microbiology. Sixth edition. Washington DC.USA: American Society for Microbiology; 1995. 